\title{
What questions do patients undergoing lower extremity joint replacement surgery have?
}

\author{
Alex Macario*1, Peter Schilling ${ }^{1}$, Richard Rubio ${ }^{1}$, Amandeep Bhalla ${ }^{2}$ and \\ Stuart Goodman ${ }^{3}$
}

Address: ${ }^{1}$ Departments of Anesthesia and Health Research \& Policy, Stanford University School of Medicine, Stanford, California, USA, ${ }^{2}$ University of Pennsylvania, Philadelphia, Pennsylvania, USA and ${ }^{3}$ Department of Orthopedic Surgery, Stanford University School of Medicine, Stanford, California, USA

Email: Alex Macario* - amaca@stanford.edu; Peter Schilling - schillin@Stanford.EDU; Richard Rubio - ricrubio@Stanford.EDU; Amandeep Bhalla - bhalla2@sas.upenn.edu; Stuart Goodman - goodbone@stanford.edu

* Corresponding author

Published: 24 June 2003

BMC Health Services Research 2003, 3:I I
Received: 04 October 2002

Accepted: 24 June 2003

This article is available from: http://www.biomedcentral.com/I472-6963/3/II

(C) 2003 Macario et al; licensee BioMed Central Ltd. This is an Open Access article: verbatim copying and redistribution of this article are permitted in all media for any purpose, provided this notice is preserved along with the article's original URL.

\begin{abstract}
Background: The value of the Internet to deliver preoperative education would increase if there was variability in questions patients want answered. This study's goal was to have patients consulting an orthopedic surgeon about undergoing either a total hip arthroplasty (THA) or a total knee arthroplasty (TKA) rate the importance of different questions concerning their care.

Methods: We assembled questions patients might have about joint replacement surgery by analyzing the literature and querying a pilot group of patients and surgeons. Twenty-nine patients considering undergoing THA and 19 patients considering TKR completed a written survey asking them to rate 30 different questions, with a 5 point Likert scale from I (least important) - 5 (most important).

Results: For patients considering THA or TKR, the 4 highest rated questions were: Will the surgery affect my abilities to care for myself?, Am I going to need physical therapy?, How mobile will I be after my surgery?, When will I be able to walk normally again? The mean percentage disagreement was $42 \%$ for questions answered by TKR patients and $47 \%$ for the THA group. Some patients gave a high rating to questions lowly rated by the rest of the group.

Conclusions: Although there was enough agreement to define a core set of questions that should be addressed with most patients considering THA or TKA, some of the remaining questions were also highly important to some patients. The Web may offer a flexible medium for accommodating this large variety of information needs.
\end{abstract}

\section{Background}

Providing information tailored to the specific needs of a patient permits informed decision-making.[1] However, patient participation in health care decision-making may be compromised if physicians rely solely on verbal communication to inform patients. Patients may forget or misunderstand spoken information. Or, they may not get all their questions answered, leaving them less able to 
make an informed decision. And, there may be substantial variation in the quantity and nature of information given to patients preoperatively.[2]

Patient well-being may be improved by providing an educational pamphlet, video, or audiotape preoperatively. $[3,4]$ The Internet has become an increasingly important medium for patients seeking a wide variety of health information.[5-7] Even though the quality of the information on the Web needs to improve, medical information on a web site can be updated at low cost.[8-10] Alternatively, patients can be sent customized educational information via e-mail.

The value of the Internet to deliver medical information to patients having surgery would especially increase if a lot of variability existed in the questions that patients want answered preoperatively. This is because unlike traditional patient information tools (such as a brochure or a video where only a limited amount of preoperative information can be provided), a Web site could be structured to address the particular questions an individual patient might have.

Our hypothesis was that a small group of questions is not sufficient to meet all the preoperative information needs of a particular surgical patient. We chose to study patients considering undergoing total hip arthroplasty (THA) or total knee arthroplasty (TKA) because we are formalizing a patient education program for these patients. THA and TKA also deserve study as they are commonly performed, and provide substantial improvements in quality of life due to reduced pain and greater mobility.[11-14]

To date, however, there are few data on the particular information needs of patients considering joint replacement surgery, and which of these needs deserves highest priority.[15] A study of a broad range of patient and procedures found that preoperative concerns include: pain they might experience afterwards (57\% of patients), whether the surgery would improve their condition $(51 \%)$, and whether they would fully recover from the surgery (42\%).[16] For TKA or THA, semi-structured interviews of German patients found that they were more interested in information about the operation and recovery (43\% each) than about risks (33\%).[17]

The goal of this study was to have patients considering undergoing either total hip THA or TKA rate the importance of different questions concerning their care. Then, the optimal method of communicating these information needs can be studied.

\section{Methods}

The Stanford University Medical Center (SUMC) Human Subjects Committee approved this study.

\section{Generation of question items for survey instrument}

We assembled an initial list of questions that might be important to patients having joint replacement surgery through informal discussions between the lead author and 2 patients scheduled to have THA, 2 patients scheduled to have TKA, 2 health care researchers, and 2 orthopaedic surgeons. The list produced by these individuals was augmented by analyzing the results of a computerized literature search (MEDLINE) for 1989-2001 using the following terms: "patient information, information needs, TKA, THA." This search yielded 56 relevant published articles (a sample of these studies is included in the references) which were read by the lead author to generate other potential questions patients might have. For example, one study found that pain immediately after surgery, length of recovery, ability to walk, and ability to return to recreational activities were the 4 most important concerns of patients undergoing THA or TKA.[18]

The authors then qualitatively edited (e.g., similar questions were eliminated) the list to a total of 30 items. The two main criteria used to determine this final list were to ensure that significant elements of care were included, and to keep the number of questions low enough so as not to make the survey take longer than 12 minutes to complete. Each question expressed one idea and no question was phrased in a negative tense (i.e., not or neither). The survey instrument was written at a sixth grade reading level, as assessed by the Flesch-Kincaid method.

The final written questionnaire instrument was organized in three parts. The first was the informed consent. The second asked for basic demographic data. The third part instructed patients to judge the importance of addressing each of 30 questions with a 5 point Likert scale from 1 (least important) - 5 (most important).

Study participants were given the following instructions: "Please rate how important it is to you that these questions be answered fully." The questionnaire also allowed patients to write in any other questions that they might have. Pilot testing suggested that completion of the survey required less than 15 minutes of the patient's time. The actual survey is available from the authors.

\section{Patients}

SUMC is a 660 bed, university affiliated, tertiary care medical center with 29,000 surgical cases/year. Patients greater than 18 years of age seen by two orthopaedic surgeons in the Stanford Orthopedic Clinic for evaluation for hip or knee replacement surgery were eligible to participate. 
Table I: Characteristics of patients

\begin{tabular}{|c|c|c|c|c|}
\hline & Stanford THA (N = II patients) & Stanford TKA ( $\mathrm{N}=10$ patients $)$ & $\begin{array}{l}\text { Southern California THA } \\
\text { ( } N=18 \text { patients })\end{array}$ & $\begin{array}{c}\text { Southern California TKA } \\
(N=9 \text { patients })\end{array}$ \\
\hline Mean Age (SD) & $53(20)$ & $60(15)$ & $70(14)$ & $68(10)$ \\
\hline \# Females & $7(64 \%)$ & $8(80 \%)$ & $11(61 \%)$ & $6(67 \%)$ \\
\hline \multicolumn{5}{|l|}{ Ethnicity: } \\
\hline \# Caucasian & II (I00\%) & $4(40 \%)$ & $10(56 \%)$ & $5(56 \%)$ \\
\hline \# Hispanic & 0 & $2(20 \%)$ & $3(17 \%)$ & $\mathrm{I}(\mathrm{II \%})$ \\
\hline \# Asian/Pacific Islander & 0 & $3(30 \%)$ & $2(11 \%)$ & I (II\%) \\
\hline \# Other & 0 & I (10\%) & $3(17 \%)$ & $2(22 \%)$ \\
\hline \multicolumn{5}{|l|}{ Schooling: } \\
\hline$\leq 12$ years & $2(18 \%)$ & $3(30 \%)$ & $6(33 \%)$ & $4(44 \%)$ \\
\hline 14 years & $2(18 \%)$ & $4(40 \%)$ & $7(39 \%)$ & $2(22 \%)$ \\
\hline 16 years & $4(36 \%)$ & I (10\%) & $3(17 \%)$ & $2(22 \%)$ \\
\hline$>16$ years & $3(27 \%)$ & $2(20 \%)$ & $2(11 \%)$ & I (I I\%) \\
\hline $\begin{array}{l}\text { Previous surgery of } \\
\text { same type }\end{array}$ & $4(36 \%)$ & $\mathrm{I}(10 \%)$ & $3(17 \%)$ & $0(0 \%)$ \\
\hline
\end{tabular}

Patients unable to speak or read English or with cognitive disabilities were excluded.

During a two-month period, 25 patients (four patients refused to participate) considering undergoing joint replacement surgery were selected randomly by computer-generated tables. No announcements or fliers were used to recruit patients. After the patient's regularly scheduled office consultation, a trained research assistant approached the eligible patient, described the study, and asked if the patient was interested in participating.

If the patient agreed, he or she was asked to complete the written questionnaire. Subjects provided consent by returning the completed survey. The identity of the patient was not recorded, and no data was collected from medical records, billing records, or any clinical or research databases. The research assistant was available to assist patients completing the survey.

In order to increase sample size, we also obtained survey data from 18 knee replacement surgery patients ( 8 refused to participate) and 9 hip replacement surgery patients (5 refused to participate) seen by one orthopedic surgeon at a community practice clinic in Southern California.

\section{Data analysis}

Since the question importance data were not normally distributed, a question's importance was ranked based on medians.

We also quantified agreement (or disagreement) in responses by determining the percentage of respondents that answered each question similarly. For example, let's assume that 10 patients answered a question with 5 pos- sible answers 1 (least important) - 5 (most important)). If 6 patients answered " 4 ", and 1 patient answered " 3 , and 1 patient answered " $1 "$, and 2 patients answered " 5 ", the agreement percentage equaled $6 / 10(60 \%)$. To quantify variability in answers, we also computed the percentage of answers that were 2 or more units different than the overall median for that question. In the example above, this would equal $1 / 10(10) \%$.

\section{Results}

Patient characteristics from the two clinics were similar. (Table 1)

The survey results from the 2 clinic locations were combined because a) for $50 \%$ of the questions the median scores were equal between the 2 sites, b) the difference in medians equaled 0.5 on $10 \%$ of the questions, and c) the difference in medians equaled 1 for the remaining $40 \%$ of questions.

The following 4 questions were rated as most important with a median score of 5 by TKA and THA patients at both sites: Will the surgery affect my abilities to care for myself? Am I going to need physical therapy? How mobile will I be after my surgery? When will I be able to walk normally again? (Table 2)

However, overall there was disagreement on question importance. For example, the mean percentage disagreement among all 30 questions was $42 \%$ for TKR patients and $47 \%$ for the THA patients.

Some questions that overall had high median scores for importance were rated by a few patients to be of low importance, and vice a versa. (Tables 3 and 4) 
Table 2: Patients' overall ranking (median scores) of the importance of addressing questions regarding joint replacement surgery

\begin{tabular}{|c|c|c|}
\hline Question I (least important) - 5 (most important) & THA ( $\mathrm{N}=29$ patients $)$ & TKA ( $N=19$ patients $)$ \\
\hline Will the surgery affect my abilities to care for myself? & 5 & 5 \\
\hline Am I going to need physical therapy? & 5 & 5 \\
\hline How mobile will I be after my surgery? & 5 & 5 \\
\hline When will I be able to walk normally again? & 5 & 5 \\
\hline What are my options if I decide not to receive surgery? & 5 & 4 \\
\hline Will the surgery cause pain afterwards? & 5 & 4 \\
\hline How long will I be in the hospital? & 5 & 4 \\
\hline Is there anything I can do to eliminate pain after surgery? & 4 & 5 \\
\hline Will I receive medication to manage the pain? & 4 & 4 \\
\hline How will I use the restroom after the surgery? & 4 & 4 \\
\hline Will I have an increased chance of bleeding after surgery? & 4 & 4 \\
\hline Will I need somebody to take me to and from physical therapy? & 4 & 4 \\
\hline After surgery what is my physical therapy going to be like? & 4 & 4 \\
\hline What is the cost of my surgery and physical therapy? & 4 & 4 \\
\hline How common is the condition I have? & 4 & 4 \\
\hline How flexible is the physical therapy program after my surgery? & 4 & 4 \\
\hline Where can I get more information about my disease and ...? & 4 & 4 \\
\hline Will I see the orthopedic surgeon after the surgeon? & 4 & 4 \\
\hline How will I be able to bathe myself after the surgery? & 4 & 4 \\
\hline Will the surgery affect the way I sleep? & 4 & 4 \\
\hline How long will I be under anesthesia? & 4 & 4 \\
\hline Who will take care of me after I leave the hospital? & 4 & 3 \\
\hline Do I continue taking medications during rehabilitation? & 4 & 3 \\
\hline Are there people I can reach for more information and support? & 4 & 3 \\
\hline How many patients like me have decided to have this treatment? & 3 & 4 \\
\hline Will my surgery decision affect my social life? & 3 & 3.5 \\
\hline Will I be able to do household chores/work in the garden after? & 3 & 3.5 \\
\hline Will the physical therapy make me tired? & 3 & 3 \\
\hline Will the surgery affect my sexual functioning? & 3 & 2 \\
\hline Will my surgery decision change the way I look? & 3 & 2 \\
\hline
\end{tabular}

For example, of the 7 questions rated highest with a median of 5 by the THA group, there was at least one patient (total of 11 patients) for 6 of the 7 questions that rated it a 2 or less. Overall, for the THA group, 16\% of all the questions answered $(\mathrm{N}=30$ questions multiplied by 29 THA patients) were 2 or more units different than the overall median for that question.

For the TKA group, 25\% of all the questions answered (N $=30$ questions multiplied by 19 patients) were 2 or more units different than the overall median for that question. For example, of the 5 questions rated the lowest (median score of 3.5 or less), there was a mean of 4 (range 2-6) patients for each question that rated it a 5 .

Patients studied were also asked to list other questions that they might want answered besides those on the questionnaire. No single question was suggested by more than one respondent. (Table 5)

\section{Discussion}

We found that there was enough agreement to define a core set of questions that should be addressed with most patients considering undergoing either TKA or THA. These questions rated to be of high concern related to whether the surgery affected the patient's ability to care for themselves, whether they would need physical therapy, and how mobile (i.e., able to walk again) they would be after surgery.

However, each of the remaining questions in our survey was also judged to be of highest importance to some of the patients. Some patients wrote on the questionnaire other questions they wanted answered. This finding of variability in patient preferences is similar to other studies. For example, although avoiding nausea/vomiting and incision pain are high priorities for most patients after surgery, there is important variability on how patients rate different postoperative outcomes.[19] 
Table 3: Total hip arthroplasty - number of patients that answered for each of the five ratings

\begin{tabular}{|c|c|c|c|c|c|}
\hline \multirow[b]{2}{*}{ Question } & \multicolumn{5}{|c|}{ I (least important) -5 (most important) } \\
\hline & 1 & 2 & 3 & 4 & 5 \\
\hline Will the surgery affect my abilities to care for myself? & 0 & 2 & I & 5 & 21 \\
\hline Am I going to need physical therapy? & 0 & 1 & 5 & 3 & 20 \\
\hline How mobile will I be after my surgery? & I & 2 & 0 & 1 & 24 \\
\hline When will I be able to walk normally again? & 0 & 1 & 3 & 8 & 17 \\
\hline What are my options if I decide not to receive surgery? & 0 & 0 & 2 & 5 & 22 \\
\hline Will the surgery cause pain afterwards? & 0 & 1 & 4 & 6 & 18 \\
\hline How long will I be in the hospital? & 1 & 2 & 5 & 3 & 17 \\
\hline Is there anything I can do to eliminate pain after surgery? & 0 & 2 & 2 & 15 & 10 \\
\hline Will I receive medication to manage the pain? & 0 & 0 & 4 & 14 & 11 \\
\hline How will I use the restroom after the surgery? & 0 & 3 & 7 & 10 & 8 \\
\hline Will I have an increased chance of bleeding after surgery? & 2 & 3 & 6 & 11 & 7 \\
\hline Will I need somebody to take me to \& from physical therapy? & 0 & 3 & 9 & 10 & 7 \\
\hline After surgery what is my physical therapy going to be like? & 2 & 2 & 6 & 13 & 6 \\
\hline What is the cost of my surgery and physical therapy? & I & 2 & 6 & 13 & 6 \\
\hline How common is the condition I have? & 2 & 2 & 8 & 10 & 7 \\
\hline How flexible is the physical therapy program after surgery? & 0 & 2 & 9 & 12 & 6 \\
\hline $\begin{array}{l}\text { Where can I get more information about my disease and the potential } \\
\text { treatments available? }\end{array}$ & 0 & 4 & 7 & 12 & 6 \\
\hline Will I see the orthopedic surgeon after the surgeon? & 0 & 5 & 7 & 4 & 13 \\
\hline How will I be able to bathe myself after the surgery? & 0 & 3 & 10 & 4 & 12 \\
\hline Will the surgery affect the way I sleep? & 1 & 3 & 7 & 6 & 12 \\
\hline How long will I be under anesthesia? & 0 & 1 & 9 & 5 & 14 \\
\hline Who will take care of me after I leave the hospital? & 1 & 4 & 7 & 8 & 9 \\
\hline Do I continue taking medications during rehabilitation? & 0 & 1 & 10 & 9 & 9 \\
\hline Are there people I can reach for more information \& support? & 0 & 3 & 11 & 5 & 10 \\
\hline How many patients like me have decided to have this treatment? & 2 & 6 & 7 & 10 & 4 \\
\hline Will my surgery decision affect my social life? & 3 & 5 & 10 & 4 & 7 \\
\hline $\begin{array}{l}\text { Will I be able to do household chores or work on the garden after the } \\
\text { surgery? }\end{array}$ & 3 & 2 & II & 6 & 7 \\
\hline Will the physical therapy make me tired? & 2 & 9 & 11 & 4 & 3 \\
\hline Will the surgery affect my sexual functioning? & 3 & 3 & 11 & 5 & 7 \\
\hline Will my surgery decision change the way I look? & 4 & 3 & 10 & 5 & 7 \\
\hline
\end{tabular}

The difficulty in finding homogeneity in information needs suggests that the database of information required for education of patients undergoing joint replacement surgery cannot be reduced, because each piece of information was necessary to some. Providing core information to all patients plus education tailored to an individual patient's requests may be a way to address variability in patient information needs.

Perhaps this is one reason that Web sites have become available to deliver information to patients.[20] Increasingly, more patients have Internet access. One European study of cancer screening found that $45 \%$ of patients had home Internet access and a further $32 \%$ had web access from other sources.[21] Fifty-six percent of orthopedic patients in London had access to the web. [22] U.S. studies have found similar rates of Internet access. [23,24]
A single Web site is part of a larger net of information so that a topic not covered by one Web page may be covered by another, via links. [25] Future studies need to be designed to determine if in-depth, and evidence-based educational information available via a web site is better at accommodating preoperative patient education than paper media or videos given out in the waiting room prior to the patient seeing a surgeon. Importantly, physicians in a group would have to reach consensus on the content of "easy to understand" answers for each question. Some of the questions may be difficult to address due to lack of clear evidence.

As in most studies in health care, our subject sample depended on patients' willingness to participate and the types of patients seen in the two clinics studied. The potential for selection bias was minimized, to the extent possible by sampling a group of patients of various ages, 
Table 4: Total knee arthroplasty - number of patients that answered for each of the five ratings

\begin{tabular}{|c|c|c|c|c|c|}
\hline \multirow[b]{2}{*}{ Question } & \multicolumn{5}{|c|}{ I (least important) - 5 (most important) } \\
\hline & 1 & 2 & 3 & 4 & 5 \\
\hline Will the surgery affect my abilities to care for myself? & 0 & 1 & 1 & 7 & 10 \\
\hline Am I going to need physical therapy? & 0 & 1 & 1 & 4 & 13 \\
\hline How mobile will I be after my surgery? & I & 1 & 2 & 3 & 12 \\
\hline When will I be able to walk normally again? & I & 1 & 2 & 4 & II \\
\hline What are my options if I decide not to receive surgery? & 3 & 0 & 2 & 5 & 9 \\
\hline Will the surgery cause pain afterwards? & 1 & 1 & 4 & 7 & 6 \\
\hline How long will I be in the hospital? & I & 1 & 6 & 4 & 7 \\
\hline Is there anything I can do to eliminate pain after surgery? & I & 0 & 3 & 4 & 11 \\
\hline Will I receive medication to manage the pain? & 2 & 1 & 3 & 5 & 8 \\
\hline How will I use the restroom after the surgery? & I & 1 & 5 & 5 & 7 \\
\hline Will I have an increased chance of bleeding after surgery? & I & 0 & 6 & 7 & 5 \\
\hline Will I need somebody to take me to \& from physical therapy? & 2 & 2 & 3 & 5 & 7 \\
\hline After surgery what is my physical therapy going to be like? & 1 & 2 & 4 & 6 & 6 \\
\hline What is the cost of my surgery and physical therapy? & 2 & 1 & 5 & 5 & 6 \\
\hline How common is the condition I have? & 0 & 0 & 9 & 4 & 6 \\
\hline How flexible is the physical therapy program after surgery? & I & 2 & 5 & 5 & 6 \\
\hline $\begin{array}{l}\text { Where can I get more information about my disease and the } \\
\text { potential treatments available? }\end{array}$ & 1 & 2 & 4 & 4 & 8 \\
\hline Will I see the orthopedic surgeon after the surgeon? & 2 & 0 & 4 & 5 & 8 \\
\hline How will I be able to bathe myself after the surgery? & I & 3 & 3 & 5 & 7 \\
\hline Will the surgery affect the way I sleep? & 2 & 1 & 6 & 7 & 3 \\
\hline How long will I be under anesthesia? & 2 & 0 & 7 & 4 & 6 \\
\hline Who will take care of me after I leave the hospital? & I & 1 & 8 & 4 & 5 \\
\hline Do I continue taking medications during rehabilitation? & i & 0 & 9 & 3 & 6 \\
\hline Are there people I can reach for more information \& support? & 3 & 2 & 8 & 3 & 3 \\
\hline How many patients like me have decided to have this treatment? & 1 & 1 & 6 & 3 & 8 \\
\hline Will my surgery decision affect my social life? & 4 & 1 & 4 & 5 & 4 \\
\hline $\begin{array}{l}\text { Will I be able to do household chores or work on the garden after } \\
\text { the surgery? }\end{array}$ & 3 & 1 & 5 & 3 & 6 \\
\hline Will the physical therapy make me tired? & 5 & 2 & 5 & 2 & 4 \\
\hline Will the surgery affect my sexual functioning? & 7 & 3 & 4 & 3 & 2 \\
\hline Will my surgery decision change the way I look? & 7 & 3 & 3 & 2 & 3 \\
\hline
\end{tabular}

Table 5: Additional questions written in by the patients

\footnotetext{
How will I be able to manage severe pain?

Tell me about my prosthesis?

What is the surgeon's medical background?

Why should I have confidence in him?

Tell me about the surgery procedure

Am I seeing a film of the surgery?

What are my post surgical physical therapy options? (in home? Out patient?)

Whom do I ask about my medications for pain and inflammation?

How many of these procedures has my surgeon done?

What is the infection rate?

How long is the entire recovery period?

How much will the physical therapy after the surgery cost?

Will this surgery lead to constipation?

What is the average length of time I will need to recover my facilities?

Are there any problems I may face in full recovery?
} 
gender, ethnicities, and education levels. Since our sample were patients that were in the final decision making period about undergoing surgery, information about risks was not included in the list presented to the patients, although it did come up in the additional questions suggested by patients. Patients who are not sure whether or not they wish to undergo these treatments are likely to be more interested in knowing about the risks, than those who have already made the decision. For example, some patients mentioned a need to know about infection rates and the surgeon's qualifications and previous experience with performing this procedure. This additional information would likely be needed for patients deciding whether or not to have surgery.

\section{Conclusions}

The content and relative importance of information that patients undergoing TKA or THA need is unclear. Since the trend is for more Internet based education, we wanted to know what questions to address on our web site to provide better individualized information to patients before surgery. We found that although there was enough agreement to define a core set of questions that should be addressed with most patients deemed suitable for the procedure by the surgeon, some of the remaining questions were also considered highly important by some patients. The core set of information, therefore, would not be adequate to satisfy any one patient's essential information needs. It may be that the Web offers a flexible medium for informing patients and accommodating their variety of needs.

\section{Competing interests}

None declared.

\section{Authors' contributions}

AM developed the research question, outlined the study protocol, formulated the survey instrument, completed the analyses, and wrote the majority of the paper. PS, RR, and $\mathrm{AB}$ enrolled patients, administered the surveys to patients, and entered data in a database. SG participated in the design of the study and its coordination.

All authors read and approved the final manuscript.

\section{Acknowledgements}

PS and RR were funded in part by The Stanford Medical Scholars Program.

\section{References}

I. Davies A and Ware J: Involving consumers in quality of care assessment Health Affairs 1988, I 5:33-9.

2. Klafta J and Roizen M: Current understanding of patient's attitudes toward and preparation for anesthesia: a review Anesth Analg 1996, 83:|3|4-2|.

3. Daltroy LH, Morlino $\mathrm{Cl}$, Eaton HM, Poss R and Liang $\mathrm{MH}$ : Preoperative education for total hip and knee replacement patients Arthritis Care Res 1998, I I(6):469-78.
4. Doering S, Katzlberger F and Rumpold G et al.: Videotape preparation of patients before hip replacement surgery reduces stress Psychosom Med 2000, 62(3):365-73.

5. Cumbo A, Agre $P$ and Dougherty J et al:: Online cancer patient education: evaluating usability and content Cancer Pract 2002, I0(3): |55-6|

6. Murphy MA and Joyce WP: Information for surgical patients: implications of the World Wide Web Eur J Surg 2001, I67(10):728-33.

7. Young SP, Henderson E and Cheseldine DL et al.: Development and assessment of a World Wide Web site for systemic lupus erythematosus patient information Lupus 2002, I I (8):478-84.

8. Allen JW, Finch RJ and Coleman MG et al.: The poor quality of information about laparoscopy on the World Wide Web as indexed by popular search engines Surg Endosc 2002, I 6(I): I702.

9. Eysenbach G, Powell J, Kuss $O$ and Sa ER: Empirical studies assessing the quality of health information for consumers on the world wide web: a systematic review JAMA 2002, 2229(287):269l-700.

10. Coiera E: The Internet's challenge to health care provision BM] 1996, 3 I 2:3-4.

II. March LM, Cross MJ and Lapsley $\mathrm{H}$ et al.: Outcomes after hip or knee replacement surgery for osteoarthritis. A prospective cohort study comparing patients' quality of life before and after surgery with age-related population norms Med J Aust I999, I 7 I(5):235-8.

12. Salmon P, Hall GM, Peerbhoy D, Shenkin A and Parker C: Recovery from hip and knee arthroplasty: Patients' perspective on pain, function, quality of life, and well-being up to 6 months postoperatively Arch Phys Med Rehabil 200 I, 82(3):360-6.

13. Liang MH, Cullen KE and Larson MG et al.: Cost-effectiveness of total joint arthroplasty in osteoarthritis Arthritis Rheum 1986, 29(8):937-43

14. Riassanen $\mathrm{P}$, Aro $\mathrm{S}$, Sintonen $\mathrm{H}$, Asikainen $\mathrm{K}$, Slatis $\mathrm{P}$ and Paavolainen $P$ : Costs and cost-effectiveness in hip and knee replacements: A prospective study International Journal of Technology Assessment in Health Care 1997, I3(4):575-88.

15. Hornberger J, Habraken $\mathrm{H}$ and Bloch D: Minimum data needed on patient preferences for accurate, efficient medical decision making Med Care 1995, 33:297-310.

16. Warfield $\mathrm{CA}$ and $\mathrm{Kahn} \mathrm{CH}$ : Acute pain management. Programs in U.S: hospitals and experiences and attitudes among U.S: adults Anesthesiology 1995, 83(5): 1090-4.

17. Hoermann S, Doering S, Richter R, Walter MH and Schussler G: Patients' need for information before surgery Psychother Psychosom Med Psychol 200I, 5 I (2):56-6I.

18. Trousdale RT, McGrory BJ, Berry DJ, Becker MW and Harmsen WS: Patients' concerns prior to undergoing total hip and total knee arthroplasty Mayo Clin Proc 1999, 74( I 0):978-82.

19. Macario A, Weinger M, Carney S and Kim A: Which clinical anesthesia outcomes are important to avoid? The perspective of patients Anesth Analg 1999, 89(3):652-8.

20. Mabrey JD: Survey of patient-oriented total hip replacement information on the World Wide Web Clin Orthop 2000 , 38I:106-13.

21. Gilliam AD, Speake WJ, Scholefield $\mathrm{JH}$ and Beckingham IJ: Finding the best from the rest: evaluation of the quality of patient information on the Internet Ann R Coll Surg Engl 2003, 85(I):44-6.

22. Gupte CM, Hassan AN, McDermott ID and Thomas RD: The internet - friend or foe? A questionnaire study of orthopaedic outpatients Ann R Coll Surg Engl 2002, 84(3): I87-92.

23. Slakey DP and Nowfar S: Internet use by a multidisciplinary transplant clinic population Transplantation 2003, I 5;75(I): I557.

24. Smith-Barbaro PA, Licciardone JC, Clarke HF and Coleridge ST: Factors associated with intended use of a Web site among family practice patients J Med Internet Res 200I, 3(2): EI7.

25. Eysenbach $G$ and Köhler $C$ : How do consumers search for and appraise health information on the World-Wide-Web? BMJ 2002, 324:573-577. 


\section{Pre-publication history}

The pre-publication history for this paper can be accessed here:

http://www.biomedcentral.com/1472-6963/3/11/prepub

Publish with Bio Med Central and every scientist can read your work free of charge

"BioMed Central will be the most significant development for disseminating the results of biomedical research in our lifetime. " Sir Paul Nurse, Cancer Research UK

Your research papers will be:

- available free of charge to the entire biomedical community

- peer reviewed and published immediately upon acceptance

- cited in PubMed and archived on PubMed Central

- yours - you keep the copyright 